\title{
Expression of the plasmacytoid dendritic cell marker BDCA-2 supports a spectrum of maturation among CD4 + CD56 + hematodermic neoplasms
}

\author{
David L Jaye ${ }^{1,2}$, Cissy M Geigerman ${ }^{1}$, Marco Herling ${ }^{3}$, Karen Eastburn ${ }^{1}$, Edmund K Waller ${ }^{2}$ \\ and Dan Jones ${ }^{3}$ \\ ${ }^{1}$ Department of Pathology and Laboratory Medicine, Emory University School of Medicine, Atlanta, GA, USA; \\ ${ }^{2}$ Winship Cancer Institute, Emory University School of Medicine, Atlanta, GA, USA and ${ }^{3}$ Department of \\ Hematopathology, University of Texas MD Anderson Cancer Center, Houston, TX, USA
}

\begin{abstract}
CD4 + CD56 + hematodermic neoplasms are rare, aggressive hematopoietic malignancies usually presenting with cutaneous masses followed by a leukemic phase. The blastic morphology, CD56 expression and lack of definitive myeloid or T-cell markers initially resulted in assignment of this tumor to the NK-cell lineage. Accumulating evidence now suggests that these neoplasms represent malignant counterparts to the plasmacytoid dendritic cell. BDCA-2 is a cell surface protein whose expression is restricted to human plasmacytoid dendritic cells, in a differentiation stage-specific manner. In the current study, we assessed expression of BDCA-2 in CD4 + CD56 + hematodermic neoplasms using a new antibody reagent we developed for use in fixed tissue sections. In 10 of 19 cases of CD4+CD56 + hematodermic neoplasm, BDCA-2 immunoreactivity was detected, whereas no expression was observed in NK-lineage tumors (0 of six). Interestingly, expression of terminal deoxynucleotidyl transferase, a marker of immaturity/blast stage, was significantly and negatively correlated with BDCA-2 in CD4 + CD56 + hematodermic neoplasms whereas a positive correlation was observed between BDCA-2 and CD7. These findings demonstrate that BDCA-2 is expressed predominantly in the CD7 + subset of hematodermic neoplasms, and similar to non-neoplastic plasmacytoid dendritic cells, expression indicates a relatively more mature differentiation state. Clinical followup data confirm the aggressiveness of these tumors and suggests that BDCA-2 immunoreactivity, as identified here, may herald a significant reduction in survival.
\end{abstract}

Modern Pathology (2006) 19, 1555-1562. doi:10.1038/modpathol.3800679; published online 22 September 2006

Keywords: blastic NK-cell lymphoma; plasmacytoid dendritic cell; terminal deoxynucleotidyl transferase; CD303

$\mathrm{CD} 4+\mathrm{CD} 56+$ hematodermic neoplasms ${ }^{1-3}$ have been variably referred to as blastic NK-cell lymphomas, ${ }^{4,5}$ CD56 + blastic tumors, ${ }^{6}$ CD 4 + CD56 + lineage-negative malignancies, ${ }^{7}$ blastic type 2 dendritic cell leukemia, ${ }^{8}$ and plasmacytoid dendritic cell leukemia/lymphoma. ${ }^{9}$ CD4 + CD56 + hematodermic neoplasms are characterized by presentation as extranodal masses that are often cutaneous, with eventual leukemic dissemination and a clinically aggressive course. ${ }^{8}$ A growing body of phenotypic and functional data argues that they represent the

Correspondence: Dr DL Jaye, MD, Department of Pathology and Laboratory Medicine, Emory University School of Medicine, 615 Michael Street-105R, Atlanta, GA 30322, USA.

E-mail:dljaye@emory.edu

Received 27 June 2006; revised and accepted 1 August 2006; published online 22 September 2006 malignant counterparts to plasmacytoid dendritic cells, or their precursors. ${ }^{2,7-11}$ For example, tumors display near-uniform expression of CD4, CD123 and TCL1, HLA-DR and CD45RA which are features of non-neoplastic plasmacytoid dendritic cells at various stages of differentiation. ${ }^{12,13}$ CD4 + CD56 + hematodermic neoplasms also fail to express markers of other hematopoietic lineages such as surface or cytoplasmic CD3, CD11b, CD11c, CD13, CD14, myeloperoxidase, lysozyme, CD117, CD19, CD20, CD79a, and CD138. Unlike many CD56 + NK-cell malignancies, Epstein-Barr virus infection has not been detected in CD4 + CD56 + hematodermic neoplasms.

The few reports of functional studies of cultured cells from CD4 + CD56 + hematodermic neoplasms indicate parallels with plasmacytoid dendritic cells, including the capacity to differentiate into compe- 
tent antigen presenting cells that express co-stimulatory molecules (eg CD80, CD86) and promote antigen-specific activation of T cells. ${ }^{10} \mathrm{In}$ one study, cultured CD4 + CD56 + hematodermic neoplasm cells produced interferon- $\alpha$ in response to viral challenge ${ }^{14}$ which is a central functional property of plasmacytoid dendritic cells. However, CD4 + CD56 + hematodermic neoplasms also demonstrate a degree of immunophenotypic heterogeneity and show patterns of disease recurrence suggesting multi-lineage potential. ${ }^{15}$ For example, variable expression of CD2, CD7, CD33, CD36, and CD68 has been demonstrated and a subset of cases display terminal deoxynucleotidyl transferase (TdT) immunoreactivity. ${ }^{1,3,16-18}$

Blood dendritic cell antigen-2 (BDCA-2) is a recently described cell-surface protein that is selectively expressed on plasmacytoid dendritic cells. ${ }^{19}$ Primary amino-acid sequence homology suggests that BDCA-2 (CLEC4C, HECL, CD303) may act as a carbohydrate-binding protein of the C-type lectin family, although specific ligands have not been reported. ${ }^{20}$ Downstream functions of BDCA-2 are still under investigation, although roles in regulating interferon- $\alpha$ production and antigen uptake have been proposed based on studies employing ligating antibody as a surrogate ligand. ${ }^{20}$ Several investigators have detected BDCA-2 expression in leukemic CD4 + CD56 + hematodermic neoplasms by flow cytometric studies, principally using the mouse monoclonal anti-BDCA-2, clone AC144..$^{8,10,21-23}$ BDCA-2 expression in infiltrating CD4 + CD56 + hematodermic neoplasms is not well characterized, largely due to lack of appropriate reagents for use in archived tissue sections.

We report here the production and characterization of rabbit polyclonal antibodies that can selectively detect BDCA-2 in fixed tissue specimens. We show that CD4 + CD56 + hematodermic neoplasms display variable expression of BDCA-2, in an inverse pattern with expression of TdT. Interestingly, clinical correlation data suggest that immunoreactivity for BDCA-2 may indicate a foreshortened survival. These data support a model in which CD4+ CD56 + hematodermic neoplasms represent transformation that recapitulates different stages of plasmacytoid dendritic cell maturation and manifest different biological potentials.

\section{Materials and methods}

\section{Antibodies}

Rabbit anti-sera were raised against a synthetic peptide containing the short cytoplasmic domain of BDCA-2 (amino acids 1-20; Cocalico Biologicals). The peptide was synthesized using standard fMOC chemistry with mass spectroscopy sequence confirmation (Global Peptide Services). Specific antipeptide antibodies (anti-cytoBDCA-2) were purified by peptide affinity chromatography using the
SulfoLink kit (Pierce Chemical Co). Other antibodies included mouse monoclonal antibodies directed against BDCA-2 (AC144, Miltenyi Biotec, Auburn, CA, USA), CD2 (AB75, Novocastra, Newcastleupon-Tyne, UK), CD7 (CBC.27, DakoCytomation), CD45RA (4KB5, DakoCytomation, Carpinteria, CA, USA), CD123 (6H6, eBioscience, San Diego, CA, USA (11)), TCL1, ${ }^{11}$ and TdT (SEN28, Novocastra, Birmingham, UK).

\section{Cloning and Expression of Human BDCA-2}

A donor apheresis product was obtained from a healthy volunteer using an institutionally approved protocol. Plasmacytoid dendritic cells, totaling $5 \times 10^{6}$ were isolated using the MACS separation and monoclonal anti-BDCA-2 (Miltenyi Biotec). RNA was purified ${ }^{24}$ followed by reverse transcription using the gene specific oligonucleotide primer, BDCA-2-R (5'-CTCGAGTATG TAGATCTTCT TCATCTT-3'). Specific PCR employed primers BDCA-2-F (5'-CATATGGTGC CTGAAGAAGA GC-3') and BDCA-2-R. DNA sequencing confirmed the correct full-length coding region, ${ }^{20}$ which was subcloned into the mammalian expression vector pcDNA3.1 + (Invitrogen) to generate pFL.BDCA-2. After transfection of pFL.BDCA-2 into 293T cells, surface expression was confirmed by flow cytometry using PE-labeled monoclonal anti-BDCA-2 (Miltenyi Biotec).

\section{Immunoprecipitation}

Immunoprecipitations of Triton X-100 detergent extracts cells were performed using monoclonal anti-BDCA-2 (AC144) or control IgG and protein A beads as described. ${ }^{20}$ Precipitated material underwent sodium dodecyl sulfate (SDS)-PAGE and transfer for immunoblot detection with anti-cytoBDCA-2.

\section{Immunoblotting}

Cells and control cells were extracted into reducing SDS-sample buffer for immunoblotting. ${ }^{25}$ SDS cell extracts were subject to electrophoresis on $10 \%$ reducing SDS polyacrylamide gels followed by transfer to nitrocellulose filters. After blocking, filters were probed with anti-cyto BDCA-2 (1:200). Bound antibodies were detected with horseradish peroxidase -conjugated goat anti-rabbit IgG (Jackson Labs) and ECL substrate (Pierce Chemical Co.) for detection with X-ray film.

\section{Human Tissue Samples}

Sections of formalin-fixed, paraffin-embedded tissues were drawn from the archives of the MD Anderson Cancer Center, the Emory University 
Division of Anatomic Pathology, British Columbia Cancer Agency, University of Pennsylvania, Miami Valley Hospital, Children's Hospital of Austin, University of Nebraska Medical Center, and Geisinger Medical Center and frozen tissue samples were obtained from the Emory University Human Tissue Procurement and Banking Service, with appropriate institutional approval. Cases of CD4+ CD56 + hematodermic neoplasm required CD4 and CD56 positivity and lack of surface CD3 expression. In addition, all cases also expressed CD123 and TCL1 to some extent. Control NK cell tumors were selected at random from the MD Anderson Cancer Center archives and included Epstein-Barr virusexpressing nasal-type NK/T-cell tumors and aggressive NK-cell leukemia/lymphoma, as defined in the current World Health Organization classification of hematopoietic tumors. Median tumor cell size was recorded in relation to the nuclei of an adjacent macrophage (intermediate cell size) and the nuclear chromatin features were graded as more blastoid (fine chromatin) or mature, with prominent nucleoli.

Available patient survival data were analyzed by the Kaplan Meier method and comparisons were made using the log-rank test. ${ }^{26} P$-values were 2sided and a value $<0.01$ was accepted as significant.

\section{Immunostaining}

For immunofluorescence studies, cryostat sections were fixed, permeablized and blocked as previously. ${ }^{27}$ Co-staining utilized rabbit anti-cytoBDCA-2 (1:200) and mouse anti-BDCA-2 (1:10). Bound antibodies were detected with anti-rabbit IgG Alexa568 and anti-mouse IgG Alexa-488. Nuclei were counter-stained with Topro-3-iodide according to manufacturer's instructions (Molecular Probes). Sections were mounted and cover slipped for viewing by immunofluorescence confocal microscopy using a $\times 63$ objective as detailed previously. ${ }^{25,27}$

For immunohistochemical analysis, formalinfixed, paraffin-embedded tissue sections underwent deparaffinization and rehydration followed by antigen unmasking in $100 \mathrm{mM}$ Tris buffer, $\mathrm{pH} 10 .{ }^{28}$ For single antibody labeling experiments, after blocking, primary antibodies, including anti-cyto BDCA-2 (1:50), anti-CD123 (1:300), and anti-CD3 (1:5), were separately applied. Detection of primary antibodies with the LSAB + System for immunoperoxidase staining and standard $\mathrm{DAB}$ reaction followed the manufacturer's recommendations (DakoCytomation). In separate experiments, sections were costained with rabbit anti-cyto BDCA-2 (1:50) and mouse anti-TdT (1:50) as primary antibodies. The Envision Doublestain system (DakoCytomation) was employed to detect bound primaries with standard DAB and fast-red reactions. Sections were counterstained with hematoxylin before mounting for microscopy.
Staining was graded semi-quantitatively. For CD7, CD45RA CD123, TCL1, immunoreactivity was graded as 0 (negative), $1+$ (weak), or +2 (strong). For TdT and BDCA-2, immunoreactivity was graded on the proportion of immunoreactive tumor cells as 0 (none), $1+$ (up to $5 \%$ ), $2+$ (up to $25 \%$ ), $3+$ $(25-50 \%)$ or uniform, strong expression $(4+)$.

\section{Results}

\section{Specificity of Cyto-BDCA-2 Antibodies}

To determine whether CD4 + CD56 + hematodermic neoplasms express BDCA-2, we generated antibodies against a synthetic peptide representing the predicted short amino-terminal cytoplasmic domain of BDCA-2. Biochemical specificity was first assessed in immunoprecipitation and immunoblotting experiments. BDCA-2 was immunoprecipitated from detergent cell extracts using the commercial specific anti-BDCA-2 monoclonal antibody (AC144) as previously described by Dzionek et $a l^{20}$ Immunoprecipitates underwent SDS-PAGE and immunoblotting with cyto-BDCA-2 antibodies. Results in Figure 1a show a prominent band of the expected $\sim 37 \mathrm{kDa}$ apparent molecular weight ${ }^{20}$ detected by the cyto-BDCA-2 antibody in the specific BDCA-2 immunopreciptate, but not in the control. Bands in the control lane (CTL IP) are consistent with cross reactivity of the secondary anti-immunoglobulin detection reagent with antibody used for immunoprecipitation. SDS extracts of human 293T cell transfectants expressing full-length BDCA-2, control mock transfectant 293T cells and control adherent cell lines T-84 and CaCo2 underwent SDS-PAGE and transfer to nitrocellulose filters. As shown in Figure $1 \mathrm{~B}$, probing the filter with the cyto-BDCA-2 antibodies yielded a single broad band of $\sim 37 \mathrm{kDa}$ only for cells transfected with BDCA-2. Negligible background binding to control cell extracts

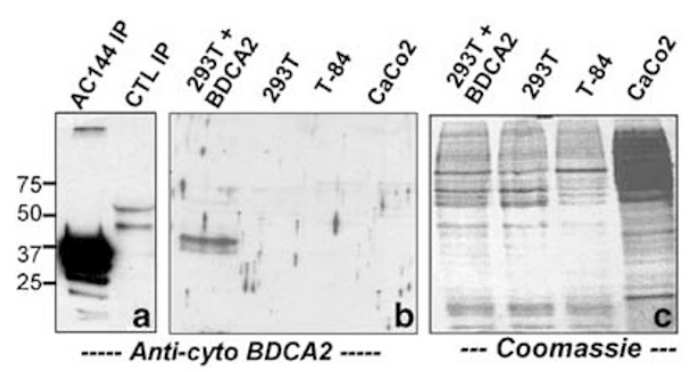

Figure 1 Biochemical selectivity of cyto-BDCA-2 antibodies. (a) Detergent extracts of BDCA-2 expressing cells underwent immunoprecipitation with anti-BDCA-2 monoclonal antibody clone AC144 (AC144 IP) or isotype control IgG (CTL IP) followed by SDS-PAGE and transfer for immunoblot detection with anti-cytoBDCA-2. ${ }^{20}$ (b) Immunoblot comparing SDS extracts of 293T cells expressing BDCA-2 (293T + BDCA-2) and control cell extracts, including mock transfectant 293T (293T), T84 and CaCo2 cells probed with cyto-BDCA-2 antibodies. (c) A Coomassie-stained replicate SDS-PAGE gel is displayed to the right. 
(mock-transfected 293T, T-84, and CaCo2) was noted. Roughly equivalent protein loading for each lane was confirmed in a replicate Coomassie-stained SDS gel displayed in Figure 1c. These data confirm the biochemical selectivity of cyto-BDCA-2 antibodies.

\section{Cyto-BDCA-2 Antibodies Detect Plasmacytoid Dendritic Cells in Fresh-Frozen and Formalin-Fixed Tissues}

Using these antibodies, we assessed the expression of BDCA-2 in hyperplastic tonsils where plasmacytoid dendritic cells are abundant. ${ }^{20}$ Detection of plasmacytoid dendritic cells using immunofluorescence microscopy on cryostat sections was compared using cyto-BDCA-2 antibodies and the well characterized monoclonal anti-BDCA-2. Colocalized membrane staining of scattered dendritic cells was noted with both the monoclonal BDCA-2 antibody (Figure 2a, green) and the rabbit cyto-BDCA-2 antibodies (Figure 2b, red) in the same cells as indicated in the merged image (Figure 2c, yellow). These immunoreactive cells were clustered in the paracortical regions as expected for plasmacytoid dendritic cells. ${ }^{20,29}$ Immunofluorescence costaining experiments for CD123 and cyto-BDCA-2 also demonstrated a similar pattern of overlapping immunoreactivity, except for added CD123 reactivity in endothelium (data not shown).

Similarly, formalin-fixed paraffin-embedded sections of tonsil were immunostained for cyto-BDCA-2 and CD123, a relatively selective marker for plas- macytoid dendritic cells in lymphoid tissue sections. ${ }^{11,30,31}$ BDCA-2 immunoreactivity was noted in clusters of cells adjacent to germinal centers (Figure 2d) that overlapped with anti-CD123 staining patterns (Figure 2e), and was concentrated in the $\mathrm{CD} 3+$ T-cell-rich interfollicular areas (Figure 2F) where plasmacytoid dendritic cells are most abundant. ${ }^{11,20,30-32}$ Therefore, cyto-BDCA-2 antibodies allow selective plasmacytoid dendritic cell staining in formalin-fixed, paraffin-embedded and fresh tissues.

\section{BDCA-2 is Expressed on a Subset of CD4 + CD56 + Hematodermic Neoplasms}

We assessed BDCA-2 expression in 19 wellcharacterized patient cases of CD4 + CD56 + hematodermic neoplasms in formalin-fixed, paraffinembedded sections including 20 specimens (Table 1) and compared them to 6 true NK-cell neoplasms. Tissue sites assessed were skin $(n=10)$, lymph node $(n=4)$, larynx $(n=1)$ and formalin-fixed, paraffinembedded bone marrow cores or clot sections $(n=5)$. Ten patients showed expression of BDCA-2 in at least $5 \%$ of tumor cells. Figures 3a (case 9) and Figure 3b (case 1) typify the expected membrane pattern of BDCA-2 immunoreactivity on tumor cells. Nine cases of CD4 + CD56 + hematodermic neoplasms were negative for BDCA-2, as were six CD56 + lymphomas of other types (4 EBV + nasaltype NK lymphomas (Figure 3c), 1 aggressive NK leukemia, 1 NK-like T-cell lymphoma).

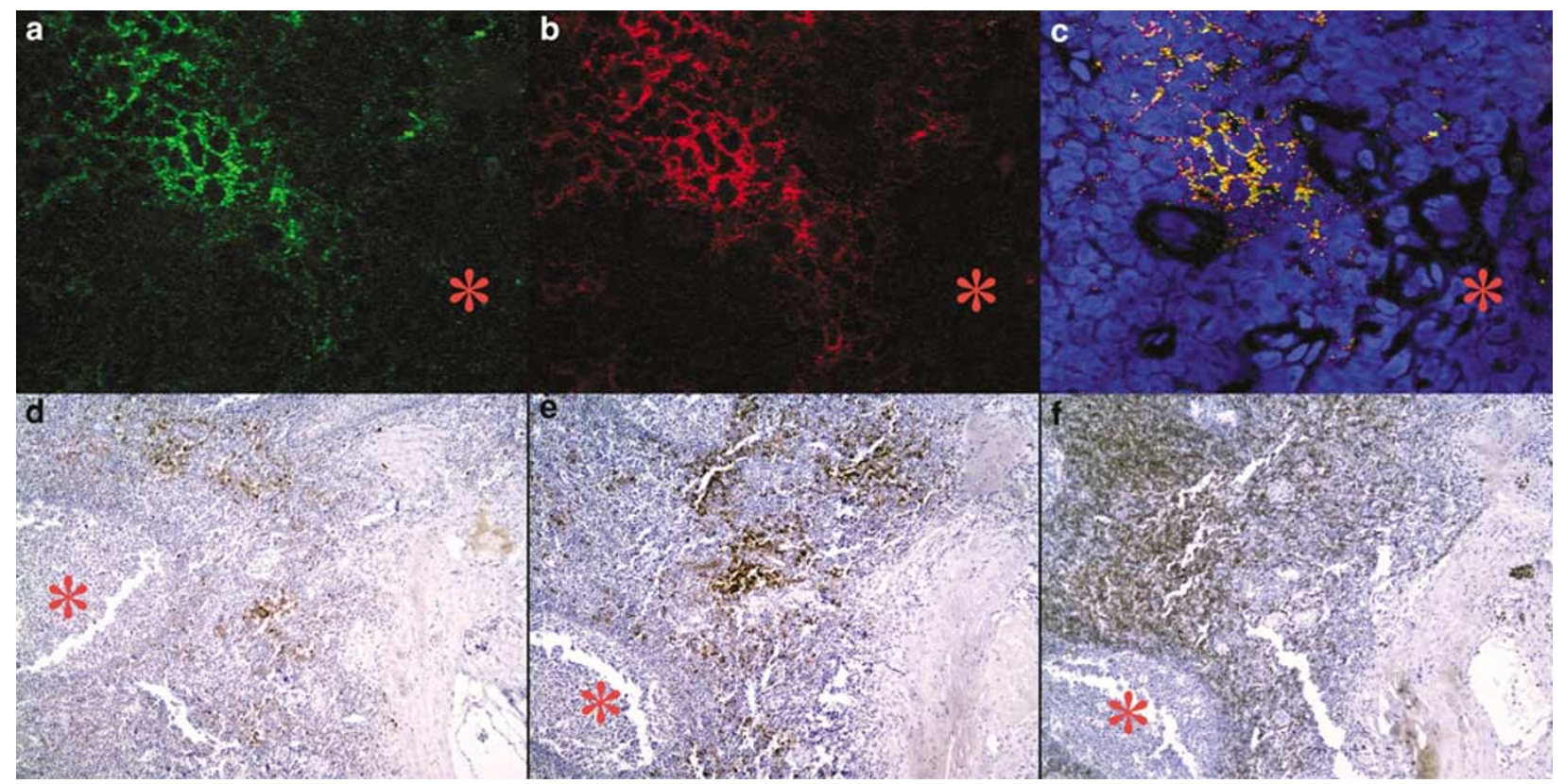

Figure 2 Reactivity of cyto-BDCA-2 antibodies with plasmacytoid dendritic cells. Confocal microscopy of cryostat sections of chronically inflamed, hyperplasic tonsil stained with the anti-BDCA-2 monoclonal antibody (a, green) and rabbit cyto-BDCA-2 antibodies (b, red). In (c), merged images display sites of co-labeling (yellow); nuclei are highlighted with Topro-3-iodide (blue). Immunohistochemical staining was performed on serial sections of formalin-fixed, paraffin-embedded human tonsil using cyto- BDCA-2 antibodies (d), anti-CD123 (e) and anti-CD3 (f). Germinal centers are indicated with red asterisks $(*)$. Original magnifications $\times 100$. 
Table 1 Pattern of BDCA-2 expression in CD4+ CD56+ hematodermic neoplasms

\begin{tabular}{|c|c|c|c|c|c|c|c|c|c|c|c|}
\hline Case & Biopsy site & Cell size/chromatin & $B D C A-2^{\mathrm{a}}$ & $T d T$ & $C D 7$ & $C D 2$ & $T C L 1$ & $C D 123$ & $C D 45 R A$ & $\begin{array}{l}\text { Follow-up } \\
\text { (days) }\end{array}$ & Outcome \\
\hline 1 & Skin & Intermediate/blastoid & $3+$ & 0 & $2+$ & 0 & $1+$ & $2+$ & 0 & & \\
\hline 2 & Bone marrow & Small/blastoid & $3+$ & 0 & $2+$ & 0 & $2+$ & $1+$ & ND & 446 & $\mathrm{DOD}^{\mathrm{c}}$ \\
\hline 3 & Bone marrow & Large/blastoid & $3+$ & 0 & $\mathrm{ND}^{\mathrm{b}}$ & ND & $1+$ & $1+$ & ND & & \\
\hline 4 & Lymph node & Large/prominent nucleoli & $2+$ & 0 & ND & ND & $1+$ & $2+$ & ND & & \\
\hline 5 & Skin & Intermediate/blastoid & $2+$ & 0 & $1+$ & $2+$ & $2+$ & $1+$ & ND & & \\
\hline 6 & Skin & Large/prominent nucleoli & $1+$ & $1+$ & $2+$ & $1+$ & $2+$ & $2+$ & $2+$ & 90 & DOD \\
\hline \multirow[t]{2}{*}{7} & Lymph node & Small/blastoid & $2+$ & $2+$ & 0 & 0 & $2+$ & $2+$ & $2+$ & & \\
\hline & $\begin{array}{l}\text { Bone marrow - } \\
\text { recurrence }\end{array}$ & Small/blastoid & 0 & $2+$ & ND & ND & $2+$ & $2+$ & ND & & \\
\hline 8 & Skin & Intermediate/blastoid & $1+$ & $2+$ & $1+$ & 0 & $1+$ & $1+$ & ND & 669 & DOD \\
\hline 9 & Lymph node & Intermediate/blastoid & $1+$ & $3+$ & $2+$ & 0 & $1+$ & $2+$ & ND & 157 & DOD \\
\hline 10 & Skin & Small/blastoid & $1+$ & $3+$ & 0 & ND & $2+$ & $2+$ & $2+$ & 360 & $\mathrm{AWD}^{\mathrm{d}}$ \\
\hline 11 & Skin & Large/blastoid & 0 & $1+$ & 0 & $2+$ & $2+$ & $1+$ & 0 & 720 & $\mathrm{CR}^{\mathrm{e}}$ \\
\hline 12 & Skin & Large/prominent nucleoli & 0 & $1+$ & 0 & ND & $2+$ & $2+$ & ND & & \\
\hline 13 & Lymph node & Intermediate/blastoid & 0 & $2+$ & 0 & $2+$ & $2+$ & $1+$ & $2+$ & 2082 & AWD \\
\hline 14 & Larynx & Intermediate/blastoid & 0 & $2+$ & ND & $2+$ & $2+$ & $1+$ & 0 & 1074 & CR s/p BMT \\
\hline 15 & Bone marrow & Small/blastoid & 0 & $2+$ & 0 & 0 & $2+$ & $2+$ & $2+$ & & \\
\hline 16 & Skin & Small/blastoid & 0 & $2+$ & 0 & 0 & $2+$ & $2+$ & $2+$ & 853 & DOD \\
\hline 17 & Bone marrow & Intermediate/blastoid & 0 & $3+$ & 0 & $2+$ & $1+$ & $2+$ & ND & 1157 & DOD \\
\hline 18 & Skin & Intermediate/blastoid & 0 & $4+$ & $2+$ & $2+$ & $1+$ & $2+$ & ND & 1070 & CR s/p chemo ${ }^{8}$ \\
\hline 19 & Skin & Intermediate/blastoid & 0 & $4+$ & ND & ND & $1+$ & $1+$ & ND & 655 & DOD \\
\hline
\end{tabular}

${ }^{a}$ CD7, CD45RA CD123, and TCL1 immunoreactivity was graded as 0 (negative), $1+$ (weak) or +2 (strong). TdT and BDCA-2 immunoreactivity was graded on the proportion of immunoreactive tumor cells as 0 (none), $1+$ (up to $5 \%$ ), $2+$ (up to $25 \%$ ), $3+(25-50 \%$ ), or uniform, strong expression (4+).

${ }^{\mathrm{b}} \mathrm{ND}=$ testing not done.

${ }^{\mathrm{C}} \mathrm{DOD}=$ dead of disease.

${ }^{\mathrm{d}} \mathrm{AWD}=$ alive with disease.

${ }^{\mathrm{e}} \mathrm{CR}=$ complete remission

$\mathrm{f}_{\mathrm{BMT}}=$ bone marrow transplantation

$\mathrm{g}_{\text {chemo }}=$ chemotherapy.

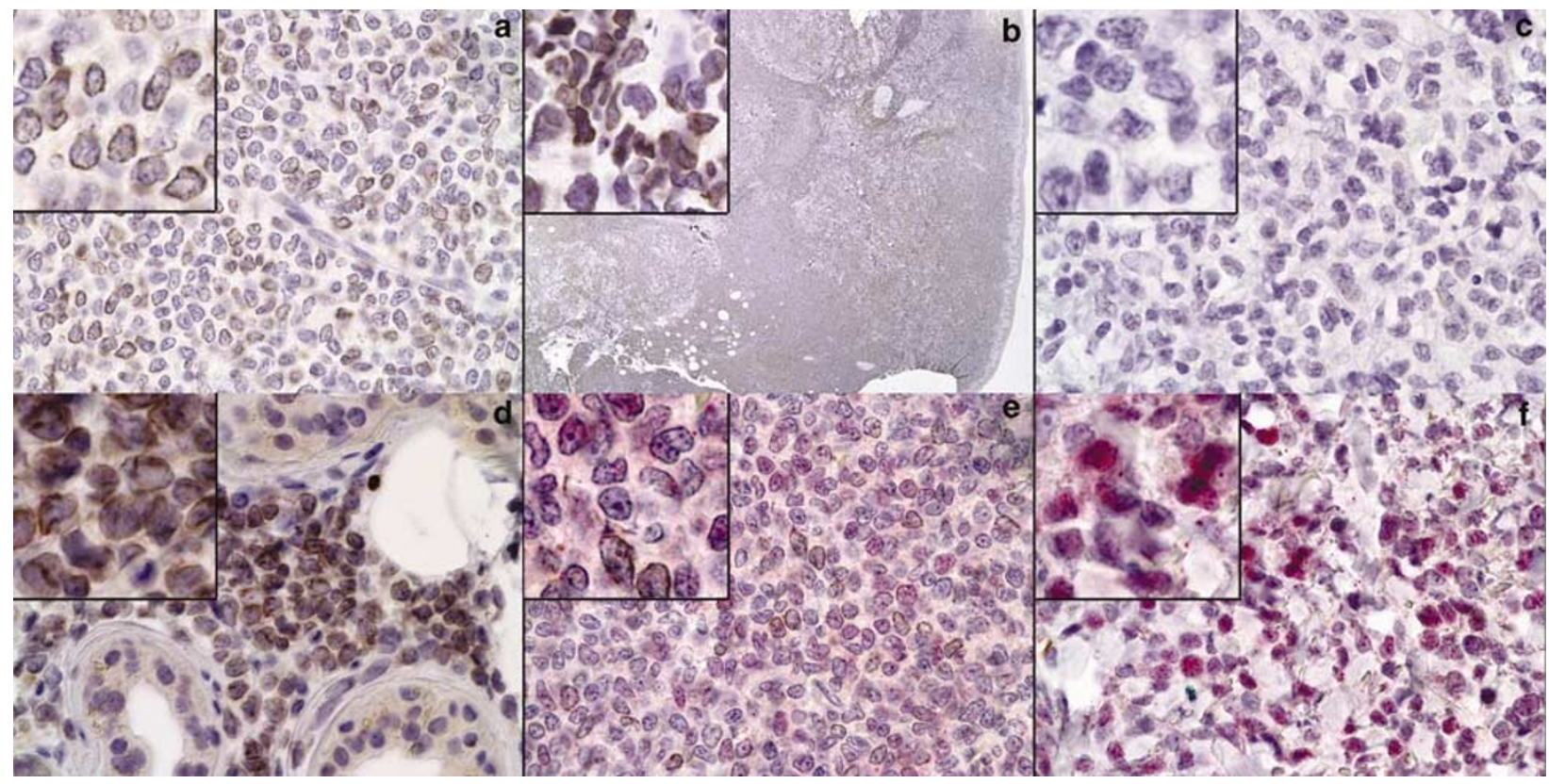

Figure 3 Reactivity of cyto-BDCA-2 antibodies with CD4 + CD56 + hematodermic neoplasm. Immunohistochemical staining of formalinfixed, paraffin-embedded sections of tumors using the anti-cyto BDCA-2 antibodies including CD4 + CD56 + hematodermic neoplasms (a, case 9; b, case 1) and a CD56 + nasal type NK/T cell lymphoma (c); original magnification $\times 600(\mathbf{a}, \mathbf{c}$, and insets a-c) $\times 20(\mathbf{b})$. Dual immunostaining of three cases of CD4 + CD56 + hematodermic neoplasm, specified in the text, with cyto-BDCA-2 antibodies (brown) and an anti-TdT antibody (red); original magnifications $\times 600$.

We assessed whether statistically significant correlations $(P<0.02)$ existed between BDCA-2 expression and others markers of CD4+CD56+ hematodermic neoplasms. BDCA-2 immunoreactivity correlated negatively with $\mathrm{TdT}$ expression (corrected Spearman rank correlation $r=-0.68$ ). 
By contrast, BDCA-2 and CD7 expression were positively correlated (corrected Spearman rank correlation $r=0.61$ ) for the 14 cases scored. Indeed, in case 7, we observed loss of both CD7 and BDCA-2 reactivity upon tumor recurrence. Double-immunostaining with BDCA-2 and TdT yielded similar results to those of individual stains as exemplified by results in Figure 3d-f. In Figure 3d, strong BDCA-2 immunoreactivity is accompanied by negligible TdT reactivity (case 5), whereas a tumor manifesting intense TdT nuclear reactivity shows a paucity of anti-BDCA-2 reactivity in Figure $3 f$ (case 18). A case displaying intermediate staining for both TdT and BDCA-2 is presented in Figure 3e (case 7). No correlations were apparent between BDCA-2 immunoreactivity and level of expression of CD2, CD123, TCL1, or the site of biopsy, tumor cell size, and nuclear features (ie blastoid/fine chromatin versus presence of prominent nucleoli).

Lastly, we asked if there were survival differences that correlate with BDCA-2 immunoreactivity. The follow-up data available for 12/19 cases are shown in Table 1 and include the length of follow-up and status at that time as categorized as either complete remission, alive with disease, or dead of disease. Follow-up time ranged from 90 to 2082 days. Kaplan Meier survival curves displayed in Figure 4 demonstrate statistically significant differences in overall survival (upper panel) and disease-free survival (lower panel) between the BDCA-2 positive and negative groups. Median overall survival and disease-free survival were approximately 1160 days for the BDCA-2 negative group. By contrast, median overall survival and disease-free survival were merely 450 days and 360 days, respectively, for the BDCA-2 positive cases.

\section{Discussion}

Here, we show that the plasmacytoid dendritic cellselective marker BDCA-2 is detectable in approximately $50 \%$ of formalin-fixed CD4 + CD56 + hematodermic neoplasms, using a new antibody reagent. These results support the previous findings of BDCA-2 detected by flow cytometry in the leukemic phase of hematodermic neoplasms ${ }^{8,10,21-23}$ and by frozen section immunostaining in very small number of cases. ${ }^{33}$ Our data suggest a greater degree of heterogeneity of expression in hematodermic neoplasms in tissues. Given the decreased sensitivity of detection for most epitopes in formalin-fixed tissues, it is not possible to be certain if BDCA-2 expression is below the limits of detection or completely negative in many cases. Nonetheless, the result adds to the growing number of observations suggesting that CD4 + CD56 + hematodermic neoplasms are an entity with considerable immunophenotypic and functional plasticity. Indeed, some CD4 + CD56 + hematodermic neoplasms recur
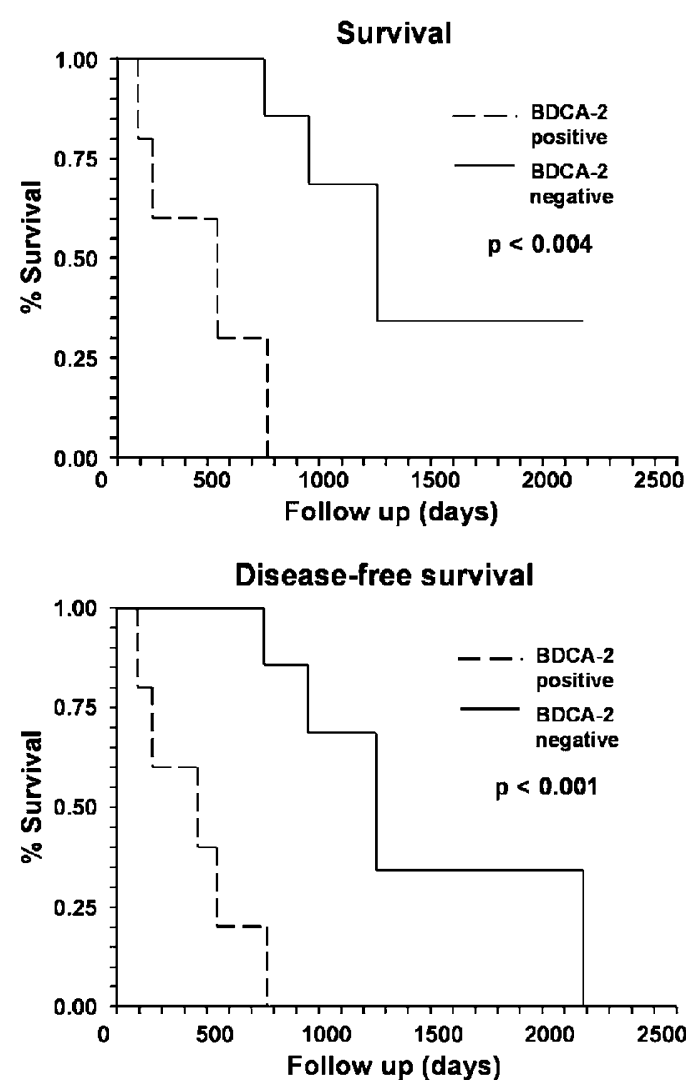

Figure 4 Kaplan-Meier estimates of patients with CD4 + CD56 + hematodermic neoplasm. Overall survival and disease-free survival of patients with BDCA-2 positive (dotted lines) and negative (straight lines) tumors as detected immunohistochemically are displayed.

following treatment as tumors with unequivocal monocytic or myelomonocytic differentiation. ${ }^{15}$

Our novel observation that there is an inverse correlation between TdT and BDCA-2 expression suggests a model for understanding this plasticity. In non-neoplastic plasmacytoid dendritic cells, BDCA2 is regulated as a maturation marker, with increased expression as plasmacytoid dendritic cell precursors mature and differentiate in the bone marrow and blood. Flow cytometric analysis of bone marrow by Dzionek et $a^{34}$ argues that cell surface BDCA-2 increases incrementally as plasmacytoid dendritic cells differentiate from CD34 + precursors. Thus, the BDCA-2-positive subset of CD4 + CD56 + hematodermic neoplasms may represent a tumor recapitulating a more mature plasmacytoid dendritic cell stage and conversely, the TdT + subset may represent a less mature, precursor plasmacytoid dendritic cell leukemia/lymphoma. Interestingly, expression of BDCA-2 and CD7 on plasmacytoid dendritic cells appears independently to identify stages of differentiation manifesting distinct immunologic capacities. ${ }^{13,19}$ If this paradigm applies as well to CD4+ CD56 + hematodermic neoplasms, the BDCA-2/CD7 positive and negative subsets may similarly reflect functional plasticity that could impact biologic 
behavior. The survival data presented, though of limited scope, are consistent with this possibility, suggesting that BDCA-2 immunoreactivity, as demonstrated here, may impart significantly worse overall and disease-free survivals for cases of CD4 + CD56 + hematodermic neoplasms. In accord with our results, Bekkenk et $a 1^{17}$ analyzed a series of 63 blastic NK cell lymphomas, including cases taken from the literature, and reported that TdT expression served as an independent positive prognostic factor.

How might the BDCA-2-positive, TdT-negative phenotype confer a worse prognosis for CD4+ CD56 + hematodermic neoplasms than the converse BDCA-2-negative, TdT-positive phenotype? One reason for the worse prognosis of the BDCA-2positive subset may simply relate to potential for reduced response to therapies, which are often mixed acute myeloid leukemia and acute lymphoblastic lymphoma regimens. For example, such reduced response by 'more mature' BDCA-2-positive, TdT-negative tumors might reflect a marginally lower growth fraction compared to the TdT-positive subset. A more interesting speculation may be drawn from observations of Chaperot et $a 1^{10}$ who demonstrated that cultured leukemic cells of CD4+ CD56 + hematodermic neoplasms can manifest plasmacytoid dendritic cell-like immunologic properties, including upregulation of costimulatory molecules and antigen presentation to $\mathrm{T}$ cells. The ability of BDCA-2 expressing plasmacytoid dendritic cells to induce Th2 immunity and the association of donor plasmacytoid dendritic cells with decreased allo-reactivity of donor T-cells after allogeneic bone marrow transplantation ${ }^{26}$ suggest the hypothesis that the $\mathrm{CD} 4+\mathrm{CD} 56+$ malignant counterpart of plasmacytoid dendritic cell may have capacity for antigen presentation and ability to induce Th2 and anergic immune responses in vivo. In short, these properties could yield decreased antitumor immune responses in patients with BDCA-2positive, TdT-negative 'more mature' tumors that are induced by the tumor itself. By contrast, 'less mature', BDCA-2-negative, Tdt-positive tumors may represent neoplasms that, like early precursors of plasmacytoid dendritic cells, are significantly less potent at modifying immune responses, and thus more susceptible to anti-tumor immune activity. In future studies, it will be important to corroborate and expand the findings reported here and to determine if these antigen expression patterns are stable over the course of disease and whether and how functional plasticity in CD4 + CD56 + hematodermic neoplasms may impact biologic behavior and prognosis. The new BDCA-2 reagent reported here will likely aid in these endeavors.

\section{Acknowledgements}

We thank Drs Mukesh Chhanabhai (British Columbia Cancer Agency), Shereen Gheith (University of
Pennsylvania), Atef Shrit (Miami Valley Hospital), Amelia Vendrell (Children's Hospital of Austin), Dennis Weisenberger (University of Nebraska Medical Center) and Kai Zhang (Geisinger Medical Center) for contribution of case material. We are indebted to Ross Fuller and Adil Akyildiz for expert technical assistance, to Drs Shiyong Li and Diane Farhi for critical reading of the manuscript, and to Drs CS Moreno and Karen Wasilewski for assistance with statistical analysis. This work was supported in part by grants from the NIH DK60647 and DK064399 (DLJ) and the University Research Committee of Emory University (DLJ) and the Leukemia and Lymphoma Society (EKW).

\section{References}

1 Petrella T, Bagot M, Willemze R, et al. Blastic NK-cell lymphomas (agranular CD4+CD56+ hematodermic neoplasms): a review. Am J Clin Pathol 2005;123: 662-675.

2 Petrella T, Meijer CJ, Dalac S, et al. TCL1 and CLA expression in agranular CD4/CD56 hematodermic neoplasms (blastic NK-cell lymphomas) and leukemia cutis. Am J Clin Pathol 2004;122:307-313.

3 Willemze R, Jaffe ES, Burg G, et al. WHO-EORTC classification for cutaneous lymphomas. Blood 2005; 105:3768-3785.

4 DiGiuseppe JA, Louie DC, Williams JE, et al. Blastic natural killer cell leukemia/lymphoma: a clinicopathologic study. Am J Surg Pathol 1997;21:1223-1230.

5 Chan JKC, Jaffe ES, Ralfkiaer E. Blastic NK-cell lymphoma. In: Jaffe ES, Harris NL, Stein H, Vardiman JW (eds). World Health Organization Classification of Tumours: Pathology and Genetics of Tumours of the Haemotopoietic and Lymphoid Tissues. Lyon: IARC Press, 2001, pp. 214-215.

6 Suzuki R, Nakamura S, Suzumiya J, et al. Blastic natural killer cell lymphoma/leukemia (CD56-positive blastic tumor): prognostication and categorization according to anatomic sites of involvement. Cancer 2005;104:1022-1031.

7 Reichard KK, Burks EJ, Foucar MK, et al. CD4(+) CD56(+) lineage-negative malignancies are rare tumors of plasmacytoid dendritic cells. Am J Surg Pathol 2005;29:1274-1283.

8 Feuillard J, Jacob MC, Valensi F, et al. Clinical and biologic features of CD4(+)CD56(+) malignancies. Blood 2002;99:1556-1563.

9 Chaperot L, Bendriss N, Manches O, et al. Identification of a leukemic counterpart of the plasmacytoid dendritic cells. Blood 2001;97:3210-3217.

10 Chaperot L, Perrot I, Jacob MC, et al. Leukemic plasmacytoid dendritic cells share phenotypic and functional features with their normal counterparts. Eur J Immunol 2004;34:418-426.

11 Herling M, Teitell MA, Shen RR, et al. TCL1 expression in plasmacytoid dendritic cells (DC2 s) and the related CD4+ CD56+ blastic tumors of skin. Blood 2003;101:5007-5009.

12 Petrella T, Comeau MR, Maynadie M, et al. 'Agranular CD4+ CD56+ hematodermic neoplasm' (blastic NK-cell lymphoma) originates from a population of CD56+ precursor cells related to plasmacytoid monocytes. Am J Surg Pathol 2002;26:852-862. 
13 Comeau MR, Van der Vuurst de Vries AR, Maliszewski $\mathrm{CR}$, et al. CD123bright plasmacytoid predendritic cells: progenitors undergoing cell fate conversion? J Immunol 2002;169:75-83.

14 Maeda T, Murata K, Fukushima T, et al. A novel plasmacytoid dendritic cell line, CAL-1, established from a patient with blastic natural killer cell lymphoma. Int J Hematol 2005;81:148-154.

15 Khoury JD, Medeiros LJ, Manning JT, et al. CD56(+) $\mathrm{TdT}(+)$ blastic natural killer cell tumor of the skin: a primitive systemic malignancy related to myelomonocytic leukemia. Cancer 2002;94:2401-2408.

16 Karube K, Ohshima K, Tsuchiya T, et al. Non-B, non-T neoplasms with lymphoblast morphology: further clarification and classification. Am J Surg Pathol 2003;27:1366-1374.

17 Bekkenk MW, Jansen PM, Meijer CJ, et al. CD56+ hematological neoplasms presenting in the skin: a retrospective analysis of 23 new cases and 130 cases from the literature. Ann Oncol 2004;15:1097-1108.

18 Massone C, Chott A, Metze D, et al. Subcutaneous, blastic natural killer (NK), NK/T-cell, and other cytotoxic lymphomas of the skin: a morphologic, immunophenotypic, and molecular study of 50 patients. Am J Surg Pathol 2004;28:719-735.

19 Dzionek A, Fuchs A, Schmidt P, et al. BDCA-2, BDCA3 , and BDCA-4: three markers for distinct subsets of dendritic cells in human peripheral blood. J Immunol 2000;165:6037-6046.

20 Dzionek A, Sohma Y, Nagafune J, et al. BDCA-2, a novel plasmacytoid dendritic cell-specific type II Ctype lectin, mediates antigen capture and is a potent inhibitor of interferon alpha/beta induction. J Exp Med 2001;194:1823-1834.

21 Anargyrou K, Paterakis G, Boutsis D, et al. An unusual case of CD4+ CD7+ CD56+ acute leukemia with overlapping features of type 2 dendritic cell (DC2) and myeloid/NK cell precursor acute leukemia. Eur J Haematol 2003;71:294-298.

22 Bueno C, Almeida J, Lucio $\mathrm{P}$, et al. Incidence and characteristics of CD4(+)/HLA DRhi dendritic cell malignancies. Haematologica 2004;89:58-69.

23 Jacob MC, Chaperot L, Mossuz P, et al. CD4+ CD56+ lineage negative malignancies: a new entity developed from malignant early plasmacytoid dendritic cells. Haematologica 2003;88:941-955.

24 Fujita N, Jaye DL, Kajita M, et al. MTA3, a Mi-2/NuRD complex subunit, regulates an invasive growth pathway in breast cancer. Cell 2003;113:207-219.

25 Jaye DL, Geigerman CM, Fuller RE, et al. Direct fluorochrome labeling of phage display library clones for studying binding specificities: applications in flow cytometry and fluorescence microscopy. J Immunol Methods 2004;295:119-127.

26 Waller EK, Rosenthal $\mathrm{H}$, Jones $\mathrm{TW}$, et al. Larger numbers of CD4(bright) dendritic cells in donor bone marrow are associated with increased relapse after allogeneic bone marrow transplantation. Blood 2001;97:2948-2956.

27 Fujita N, Jaye DL, Geigerman C, et al. MTA3 and the $\mathrm{Mi}-2 / \mathrm{NuRD}$ complex regulate cell fate during $\mathrm{B}$ lymphocyte differentiation. Cell 2004;119:75-86.

28 Jaye DL, Nolte FS, Mazzucchelli L, et al. Use of realtime polymerase chain reaction to identify cell- and tissue-type-selective peptides by phage display. Am J Pathol 2003;162:1419-1429.

29 Cella M, Jarrossay D, Facchetti F, et al. Plasmacytoid monocytes migrate to inflamed lymph nodes and produce large amounts of type I interferon. Nat Med 1999;5:919-923.

30 Summers KL, Hock BD, McKenzie JL, et al. Phenotypic characterization of five dendritic cell subsets in human tonsils. Am J Pathol 2001;159:285-295.

31 Cella M, Jarrossay D, Facchetti F, et al. Plasmacytoid monocytes migrate to inflamed lymph nodes and produce large amounts of type I interferon. Nat Med 1999;5:919-923.

32 Liu YJ. IPC: professional type 1 interferon-producing cells and plasmacytoid dendritic cell precursors. Annu Rev Immunol 2005;23:275-306.

33 Urosevic M, Conrad C, Kamarashev J, et al. CD4+CD56+ hematodermic neoplasms bear a plasmacytoid dendritic cell phenotype. Hum Pathol 2005; 36:1020-1024.

34 Dzionek A, Inagaki Y, Okawa K, et al. Plasmacytoid dendritic cells: from specific surface markers to specific cellular functions. Hum Immunol 2002;63: 1133-1148. 